\title{
New evidence for liver venous deprivation: safety and feasibility for extended liver resections
}

\author{
Jiang Zhang ${ }^{1,2}$, Christian J. Steib ${ }^{1}$ \\ ${ }^{1}$ Department of Medicine II, University Hospital, Liver Centre Munich, LMU Munich, Germany; ${ }^{2}$ Department of Liver Surgery and Liver \\ Transplantation Center, Ren Ji Hospital, School of Medicine, Shanghai Jiao Tong University, Shanghai, China \\ Correspondence to: Christian J. Steib, MD. Department of Medicine II, University Hospital, LMU Munich, Marchioninistrasse 15, 81377 Munich, \\ Germany. Email: christian.steib@med.uni-muenchen.de. \\ Comment on: Panaro F, Giannone F, Riviere B, et al. Perioperative impact of liver venous deprivation compared with portal venous embolization in \\ patients undergoing right hepatectomy: preliminary results from the pioneer center. Hepatobiliary Surg Nutr 2019;8:329-37.
}

Submitted Apr 03, 2020. Accepted for publication Apr 17, 2020.

doi: $10.21037 /$ atm-20-3057

View this article at: http://dx.doi.org/10.21037/atm-20-3057

For many primary and metastatic liver tumors, hepatectomy is the treatment of choice that can effectively extend the survival time of patients. Among different factors, the expected residual liver volume after resection, also known as future liver residue (FLR), is one important aspect affecting the prognosis of patients $(1,2)$. Many patients lose the chance of liver resection due to too little FLR. Portal vein embolization (PVE) is one of the most common techniques for increasing FLR. It is usually performed percutaneously with a microguidewire entering the right hepatic vein under ultrasound guidance, a Berenstein catheter is then inserted into the portal vein with the guide wire, and portogram is performed. After 3D portography, the portal vein branches are embolized with a mixture of n-butyl cyanoacrylate and lipiodol in the appropriate ratio (3). Since the first use of PVE to increase FLR and successfully completed surgery in patients previously considered to be intolerant to liver resection, PVE has been widely investigated to increase FLR before liver resection (4).

However, patients after PVE usually need to wait 4-6 weeks before the liver is hypertrophic to a sufficient volume for safe liver resection. During this waiting time, as many as $20 \%$ of patients suffering tumor progression (mainly bilobar colorectal liver metastases) is the main disadvantage of PVE (5). Therefore, new technologies have been developed to increase FLR in a shorter period of time, including association of liver distribution and portal vein ligation (ALPPS) and liver vein deprivation (LVD). ALPPS is a surgical technique involving parenchymal transection combined with ligation of the right portal vein and branches of the portal vein before resecting the diseased liver (6), it can increase FLR in a much shorter time, but has higher mortality. LVD is mainly through the jugular vein to avoid direct puncture through the right liver and the right hepatic vein embolization is also performed in addition to the right PVE described in PVE (3). LVD could achieve faster FLR growth without affecting mortality, but as a new tool the data are limited (7).

A recent study published in the Journal of Hepatobiliary Surgery and Nutrition by Dr. Fabrizio et al. compared the FLR between 16 patients receiving PVE and 13 patients receiving LVD (8). This study included the largest number of patients in the current series of studies. They compared intraoperative, preoperative and postoperative results of LVD and PVE in patients undergoing standard right hepatectomy: no statistically significant differences were observed in the two groups in terms of intraoperative bleeding, hepatic ischemia time, operative time, postoperative biliary leakage, liver atrophy, liver necrosis and sinusoidal dilatation. In addition to clinical indicators, the secondary endpoint of the study was the evaluation of histological specimens in order to compare the morphological changes of hepatocytes and sinusoidal endothelial cells between the two groups. The frequency of hepatocyte atrophy and necrosis was slightly higher in the LVD group, but had no statistical difference. Besides, 
sinusoidal dilatation occurred in 6 and 7 patients after PVE and LVD respectively. They concluded that LVD was a promising and safe procedure for inducing rapid FRL hypertrophy, with similar mortality/morbidity during and after surgery compared to PVE. Liver regeneration depends on the stimulation of the injury and the condition of the liver parenchyma, but its molecular and cellular mechanisms have not been clearly defined. Sinusoidal cells and hepatocytes play a key role in liver regeneration while hepatocyte growth factor, transforming growth factoralpha and epidermal growth factor have been proven to be important participating factors $(2,9)$.

We want to discuss some parts of this article, which may be useful for further designing experiments in this area. Firstly, as far as we know, all articles comparing PVE and LVD are retrospective studies, so a randomized controlled trial (RCT) is needed to confirm the benefits of LVD (the authors also mentioned two ongoing RCTs, which may provide new strong evidence).

Moreover, although the hypertrophy rate of LVD is better than that of PVE at the same time, recent studies have compared the efficacy and safety of LVD and PVE at the same waiting time. For patients with malignant tumors, especially those with advanced liver cancer, early liver resection can reduce the risk of tumor progression. Therefore, further research is needed to investigate the efficacy and safety with shorter intervals after LVD. In addition, some studies have reported that the complications of ALPPS have decreased significantly with increasing operating experience and more stringent indications (5). We expect a comparative study between improved ALPPS, PVE and LVD. By choosing the most appropriate technology for different patients, this result may provide new evidence for future personalized medicine.

Finally, the study also included several Child-A patients, and the effects of LVD and PVE were similar to normal patients. Patients who require liver resection are often accompanied by different degrees of cirrhosis. Because of impaired liver function and structure, patients with severe cirrhosis may not be suitable for LVD or PVE. However, whether PVE, LVD, ALPPS, or other new technologies can benefit patients with mild or moderate fibrosis requires further research.

In summary, this study provided new evidences for the safety and efficacy of LVD through rigorous patient selection and broader comparison issues. Based on the comments shown above, future studies on the effect and safety of shorter interval LVD and including patients with mild to moderate fibrosis may be beneficial for more people.

\section{Acknowledgments}

Funding: None.

\section{Footnote}

Provenance and Peer Review: This article was commissioned and reviewed by the Academic Editor Yuhui Fan (Internal Medicine II, Klinikum rechts der Isar, Technische Universität München, Munich, Germany).

Conflicts of Interest: Both authors have completed the ICMJE uniform disclosure form (available at http://dx.doi. org/10.21037/atm-20-3057). The authors have no conflicts of interest to declare.

Ethical statement: The authors are accountable for all aspects of the work in ensuring that questions related to the accuracy or integrity of any part of the work are appropriately investigated and resolved.

Open Access Statement: This is an Open Access article distributed in accordance with the Creative Commons Attribution-NonCommercial-NoDerivs 4.0 International License (CC BY-NC-ND 4.0), which permits the noncommercial replication and distribution of the article with the strict proviso that no changes or edits are made and the original work is properly cited (including links to both the formal publication through the relevant DOI and the license). See: https://creativecommons.org/licenses/by-nc-nd/4.0/.

\section{References}

1. 34th Annual Meeting \& Pre-Conference Programs of the Society for Immunotherapy of Cancer (SITC 2019): part 2: National Harbor, MD, USA. 10 November 2019. J Immunother Cancer 2019;7:283.

2. Li D, Madoff DC. Portal vein embolization for induction of selective hepatic hypertrophy prior to major hepatectomy: rationale, techniques, outcomes and future directions. Cancer Biol Med 2016;13:426-42.

3. Hocquelet A, Sotiriadis C, Duran R, et al. Preoperative Portal Vein Embolization Alone with Biliary Drainage Compared to a Combination of Simultaneous Portal Vein, Right Hepatic Vein Embolization and Biliary Drainage in Klatskin Tumor. Cardiovasc Intervent Radiol 
2018;41:1885-91.

4. Luz JHM, Gomes FV, Coimbra E, et al. Preoperative Portal Vein Embolization in Hepatic Surgery: A Review about the Embolic Materials and Their Effects on Liver Regeneration and Outcome. Radiol Res Pract 2020;2020:9295852.

5. Kobayashi K, Yamaguchi T, Denys A, et al. Liver venous deprivation compared to portal vein embolization to induce hypertrophy of the future liver remnant before major hepatectomy: A single center experience. Surgery 2020. doi: 10.1016/j.surg.2019.12.006.

6. Kambakamba P, Linecker M, Schneider M, et al. Impact of associating liver partition and portal vein ligation for staged hepatectomy (ALPPS) on growth of colorectal liver metastases. Surgery 2018;163:311-7.

Cite this article as: Zhang J, Steib CJ. New evidence for liver venous deprivation: safety and feasibility for extended liver resections. Ann Transl Med 2020;8(19):1259. doi: 10.21037/ atm-20-3057
7. Jaeck D, Oussoultzoglou E, Rosso E, et al. A twostage hepatectomy procedure combined with portal vein embolization to achieve curative resection for initially unresectable multiple and bilobar colorectal liver metastases. Ann Surg 2004;240:1037-49; discussion 1049-51.

8. Panaro F, Giannone F, Riviere B, et al. Perioperative impact of liver venous deprivation compared with portal venous embolization in patients undergoing right hepatectomy: preliminary results from the pioneer center. Hepatobiliary Surg Nutr 2019;8:329-37.

9. Dirscherl K, Schlapfer M, Roth Z'graggen B, et al. Hypoxia sensing by hepatic stellate cells leads to VEGFdependent angiogenesis and may contribute to accelerated liver regeneration. Sci Rep 2020;10:4392. 